\title{
Technical Note and Correspondence
}

\section{Control of Uncertain Sampled-Data Systems: An Adaptive Posicast Control Approach}

\author{
Khalid Abidi, Yildiray Yildiz, and Anuradha Annaswamy
}

\begin{abstract}
This technical note proposes a discrete-time adaptive controller for the control of sampled-data systems. The design is inspired from the Adaptive Posicast Controller (APC) which was designed for time-delay systems in continuous time. Due to the performance degradation caused by digital approximation of continuous laws, together with the problem of assuming time-delays as integer multiples of sampling intervals, the benefits of APC could not be fully realized. In this technical note, these approximations/assumptions are eliminated. In addition, a disturbance observer is incorporated into the controller design which minimizes the effect of disturbances on the system. Extension to the case of uncertain input time-delay is also presented. The proposed approach is verified in simulation studies.
\end{abstract}

Index Terms-Delay systems, digital control, uncertain systems, adaptive control.

\section{INTRODUCTION}

Theoretical development and various experimental demonstrations of the Adaptive Posicast Controller (APC), which is developed for uncertain linear systems with known input delays, are presented in [1][4]. The core ideas utilized in the development of APC is extracted from the Smith Predictor [5]-[11], the finite spectrum assignment controller (FSA) [12]-[14], and their adaptive versions [15], [16]. Although APC performed considerably better than the industrial grade controllers during the experiments, the full advantages of the method could not be exploited due to 3 main reasons: The continuous control laws had to be approximated; the disturbance compensation was not explicit but relied upon the slowly varying disturbance dynamics; and the timedelay values were assumed to be integer multiples of the sampling interval. Although it is conventionally assumed that fast sampling is advantageous during digital approximations, it is shown in [12] that, as the sampling frequency increases, the phase margin of the FSA controller decreases. (A remedy is provided in [13].) APC, utilizing FSA structure, suffers from the same issue.

In this technical note, the above mentioned approximations/assumptions are eliminated by representing the dynamics of the plant in sampled-data form with a time-delay that is a non-integer multiple of the sampling interval and designing the controller in discrete

Manuscript received December 8, 2015; revised March 28, 2016 and July 29, 2016; accepted August 11, 2016. Date of publication August 16, 2016; date of current version April 24, 2017. Recommended by Associate Editor F. Mazenc.

K. Abidi is with the Newcastle University, School of Electrical and Electronic Engineering, Newcastle Upon Tyne NE1 7RU, United Kingdom (e-mail: khalid.abidi@newcastle.ac.uk).

Y. Yildiz is with the Bilkent University, Department of Mechanical Engineering, Bilkent 06800, Ankara Turkey (e-mail: yyildiz@ bilkent.edu.tr).

A. Annaswamy is with the Massachusetts Institute of Technology, Cambridge MA 02139-4307, MA USA (e-mail: aanna@mit.edu).

Color versions of one or more of the figures in this paper are available online at http://ieeexplore.ieee.org.

Digital Object Identifier 10.1109/TAC.2016.2600627 time. In addition, a disturbance observer method is incorporated into the controller design. Finally, a rigorous stability analysis is provided.

Several methods are proposed in the literature for continuous-time control of time-delay systems. Some examples can be seen in [17]-[29]. The book [30] is also a recent contribution, demonstrating predictive feedback in time-delay systems with extensions to nonlinear systems, delay-adaptive control and actuator dynamics modeled by PDEs. In discrete time domain, many approaches exist for the adaptive control problem [31]-[34]. The contributions of the proposed approach are that 1) the controller enables the utilization of the full benefits of the APC, which, unlike many advanced controllers, is experimentally verified and shown to be performing better than industrial grade controllers, 2) an extension to the control of sampled-data systems with uncertain input time-delay case is provided, (uncertain input time-delay case is solved for the continuous time systems without approximating the time-delay in [35]), 3) a disturbance observer is incorporated into the design which minimizes the effect of disturbances and 4) the case where the delay values that are not integer multiples of the sampling interval is addressed. The approach incorporates a modified version of the disturbance observer method proposed by the author Abidi in [39]. Preliminary results of this work is presented in [36] without any stability analysis and a disturbance-free, ideal case where the timedelay is an integer multiple of the sampling interval is presented in [37] and [38].

The organization of this technical note is as follows: Section II gives the problem definition, Section III gives the controller design, Section IV gives the extension to uncertain delay case, Section V gives a simulation example and Section VI gives the conclusion.

\section{Problem Definition}

Consider a continuous-time system given as

$$
\dot{\mathbf{x}}(t)=A \mathbf{x}(t)+B(\mathbf{u}(t-\tau)+\mathbf{f}(t))
$$

where $\mathbf{x} \in \Re^{n}$ is the vector of states, $A \in \Re^{n \times n}$ is a constant uncertain state matrix, $B \in \Re^{n \times m}$ is a constant uncertain input matrix, $\mathbf{u} \in \Re^{m}$ is the vector of the control inputs, $\tau \geq 0$ is a known input time-delay and $\mathbf{f}(t)$ is a matched unmeasurable exogenous disturbance.

Assumption 1: The sampled data representation of the plant dynamics has stable zeros.

Assumption 2: There exists a known nominal input matrix $B_{n}$ such that $B=B_{n} \Lambda$ where $\Lambda \in \Re^{m \times m}$ is a constant uncertain positive definite matrix representing control failures.

Assumption 3: The disturbance $\mathbf{f}(t)$ is smooth and bounded.

The reference model is given as

$$
\dot{\mathbf{x}}_{m}(t)=A_{m} \mathbf{x}_{m}+B_{m} \mathbf{r}(t-\tau)
$$

where $A_{m} \in \Re^{n \times n}$ is a constant Hurwitz matrix, $B_{m} \in \Re^{n \times q}$ is a constant matrix and $\mathbf{r} \in \Re^{q}$ is the reference command. Note that the 
dynamics given in the reference model (2) is the best that can be achieved with any kind of control law in terms of handling the timedelay [30]. The control problem is finding a bounded control input $\mathbf{u}$ such that $\lim _{t \rightarrow \infty}\left\|\mathbf{x}_{m}(t)-\mathbf{x}(t)\right\|=0$, while keeping all the system signals bounded.

\section{Adaptive Posicast Control LaW Design for SAMPLED-DATA SYSTEMS}

\section{A. Controller Design}

Consider the system (1), if a constant $p \in Z$ is selected such that $p T<\tau<(p+1) T$, where $T$ is the sampling interval, then the sampled-data form of (1) is obtained as

$$
\mathbf{x}_{k+1}=\Phi \mathbf{x}_{k}+\Gamma_{1} \mathbf{u}_{k-p}+\Gamma_{2} \mathbf{u}_{k-p-1}+\mathbf{d}_{k},
$$

where the matrices $\Phi \in \Re^{n \times n}, \Gamma_{1} \in \Re^{n \times m}, \Gamma_{2} \in \Re^{n \times m}$ are considered uncertain and are computed using the relations

$$
\begin{aligned}
\Phi & =e^{A T}, \Gamma_{1}=\int_{0}^{(p+1) T-\tau} e^{A \sigma} d \sigma B, \text { and } \\
\Gamma_{2} & =\int_{(p+1) T-\tau}^{T} e^{A \sigma} d \sigma B,
\end{aligned}
$$

and the disturbance vector $\mathbf{d}_{\mathrm{k}}$ is computed by the relation

$$
\mathbf{d}_{k}=\int_{0}^{T} e^{A \sigma} B \mathbf{f}((k+1) T-\sigma) d \sigma .
$$

Based on the assumptions on $\mathbf{f}(t)$, the following properties are defined for $\mathbf{d}_{k}$, [39], [40]:

Property 1: The difference between two successive disturbance signals is, at most, of the order of the square of the sampling interval, i.e., $\left\|\mathbf{d}_{k}-\mathbf{d}_{k-1}\right\| \leq \Delta$ for some $\Delta \in O\left(T^{2}\right)$.

Property 2: The disturbance $\mathbf{d}_{k}$ can be represented as

$$
\mathbf{d}_{k}=\Gamma_{n}\left(L_{0} \mathbf{f}_{k}+L_{1} \dot{\mathbf{f}}_{k}\right)+O\left(T^{3}\right)=\Gamma_{n} \mathbf{w}_{k}+O\left(T^{3}\right),
$$

where $\Gamma_{n}$ is a known nominal input matrix, $\left(L_{0}, L_{1}\right) \in \Re^{m \times m}$ are uncertain matrices, $\mathbf{f}_{k}=\mathbf{f}(k T)$ and $\mathbf{w}_{k}=L_{0} \mathbf{f}_{k}+L_{1} \dot{\mathbf{f}}_{k}$.

Remark 1: If the time-delay $\tau$ is an integer multiple of the sampling-interval then $p$ is selected such that $p T=\tau$ and, consequently, $\Gamma_{1}=\int_{0}^{T} e^{A \sigma} d \sigma B$ and $\Gamma_{2}=0$.

Remark 2: For typical implementations, sampling intervals are selected as $T<1$ and, therefore, minimizing the influence of the disturbance to at most $O\left(T^{2}\right)$ is desirable.

Consider the reference model (2) in sampled-data form

$$
\mathbf{x}_{m, k+1}=\Phi_{m} \mathbf{x}_{m, k}+\Gamma_{m} \mathbf{r}_{k-p},
$$

where $\Phi_{m}$ has eigenvalues inside the unit-circle. The objective is to design a proper control law that will ensure that the system (3) will track the reference model (4) and, thereby, achieve $\lim _{k \rightarrow \infty}\left\|\mathbf{x}_{m, k}-\mathbf{x}_{k}\right\| \leq$ $\epsilon$ for some constant $\epsilon$.

To design a proper control law for the system (3), assume that the system is without uncertainty and that there exists a $\Theta \in \Re^{m \times n}$, a positive-definite $\Theta_{\gamma_{1}} \in \Re^{m \times m}$ and a positive-definite $\Theta_{\gamma_{2}} \in \Re^{m \times m}$ such that

$$
\Phi-\Gamma_{n} \Theta=\Phi_{m}, \Gamma_{1}=\Gamma_{n} \Theta_{\gamma_{1}} \text { and } \Gamma_{2}=\Gamma_{n} \Theta_{\gamma_{2}} .
$$

Consider the delay observer given as

$$
\hat{\mathbf{d}}_{k}=\mathbf{d}_{k-1}=\mathbf{x}_{k}-\Phi \mathbf{x}_{k-1}-\Gamma_{1} u_{k-p-1}-\Gamma_{2} u_{k-p-2}
$$

and select a matrix $D \in \Re^{m \times n}$ such that from Property 2 and (5) it is obtained that

$$
\mathbf{w}_{k-1}=D_{\gamma}\left(\mathbf{x}_{k}-\Phi \mathbf{x}_{k-1}\right)-\Theta_{\gamma_{1}} \mathbf{u}_{k-p-1}-\Theta_{\gamma_{2}} \mathbf{u}_{k-p-2}+O\left(T^{3}\right)
$$

where $D_{\gamma}=\left(D \Gamma_{n}\right)^{-1} D$ and $D$ is selected such that $D \Gamma_{n}$ is nonsingular. Adding and subtracting $\mathbf{d}_{k-1}$ on the right hand side of (3) and using Property 2 it is obtained that

$$
\begin{aligned}
\mathbf{x}_{k+1}= & \Phi \mathbf{x}_{k}+\Gamma_{1} \mathbf{u}_{k-p}+\Gamma_{2} \mathbf{u}_{k-p-1}+\mathbf{d}_{k}-\mathbf{d}_{k-1} \\
& +\Gamma_{n} \mathbf{w}_{k-1}+O\left(T^{3}\right) \\
= & \Phi \mathbf{x}_{k}+\Gamma_{1} \mathbf{u}_{k-p}+\Gamma_{2} \mathbf{u}_{k-p-1}+\Gamma_{n} \mathbf{w}_{k-1}+\bar{\varsigma}_{k},
\end{aligned}
$$

where $\left\|\bar{\zeta}_{k}\right\|=\left\|\mathbf{d}_{k}-\mathbf{d}_{k-1}+O\left(T^{3}\right)\right\| \leq \Delta \in O\left(T^{2}\right)$ and that, for a reasonable selection of $T, O\left(T^{2}\right) \approx O\left(T^{2}\right)+O\left(T^{3}\right)$.

Remark 3: Although $\left\|\bar{\varsigma}_{k}\right\| \in O\left(T^{2}\right)$ for a smooth disturbance, if a discontinuity occurs, $\left\|\bar{\zeta}_{k}\right\|$ becomes $O(T)$, which may negatively effect the controller performance. However, this is rare in practice and after the discontinuity the expression $\left\|\bar{\zeta}_{k}\right\| \in O\left(T^{2}\right)$ will be valid eventually.

Substituting (5) and (7) in (8) it is obtained that

$$
\begin{aligned}
\mathbf{x}_{k+1}= & \Phi_{m} \mathbf{x}_{k}+\Gamma_{n}\left(\bar{\Theta} \mathbf{x}_{k}-\Phi_{x} \mathbf{x}_{k-1}+\Theta_{\gamma_{1}} \mathbf{u}_{k-p}+\Theta_{\gamma_{21}} \mathbf{u}_{k-p-1}\right. \\
& \left.-\Theta_{\gamma_{2}} \mathbf{u}_{k-p-2}\right)+\bar{\varsigma}_{k},
\end{aligned}
$$

where $\bar{\Theta}=\Theta+D_{\gamma}, \Phi_{x}=D_{\gamma} \Phi$ and $\Theta_{\gamma_{21}}=\Theta_{\gamma_{2}}-\Theta_{\gamma_{1}}$. Performing successive substitutions on (9), it is obtained that

$$
\mathbf{x}_{k+p+1}=\Phi_{m} \mathbf{x}_{k+p}+\Gamma_{n}\left(\Theta_{x} \bar{\chi}_{k}+\Theta_{u} \bar{\xi}_{k}+\Theta_{\gamma_{1}} \mathbf{u}_{k}\right)+\bar{\delta}_{k+p},
$$

where the matrices $\Theta_{x} \in \Re^{m \times 2 n}, \Theta_{u} \in \Re^{m \times m(p+2)}$ contain the matrices $\bar{\Theta}, \Phi_{x}, \Theta_{\gamma_{1}}, \Theta_{\gamma_{2}}, \Theta_{\gamma_{21}}$, and $\bar{\chi}_{k}^{\top}=\left[\mathbf{x}_{k}^{\top} \mid \mathbf{x}_{k-1}^{\top}\right] \in \Re^{2 n}, \overline{\boldsymbol{\xi}}_{k}^{\top}=$ $\left[\mathbf{u}_{k-1}^{\top}|\cdots| \mathbf{u}_{k-p-2}^{\top}\right] \in \Re^{m(p+2)}$. The disturbance term $\bar{\delta}_{k} \in \Re^{n}$ is given as

$$
\begin{aligned}
\bar{\delta}_{k+p}= & \bar{\varsigma}_{k+p}+\Gamma_{n}\left[\bar{\Theta} \bar{\varsigma}_{k+p-1}+\left(\bar{\Theta} \Upsilon_{0}-\bar{\Phi}_{x}\right) \bar{\varsigma}_{k+p-2}+\ldots\right. \\
& \left.+\left(\bar{\Theta} \Upsilon_{p-2}-\bar{\Phi}_{x} \Upsilon_{p-3}\right) \bar{\varsigma}_{k}\right]
\end{aligned}
$$

where

$$
\Upsilon_{\eta}=\bar{\Phi}_{m}^{\eta+1}+\sum_{i=0}^{\left\lfloor\frac{\eta-1}{2}\right\rfloor}\left[\frac{(-1)^{i+1}}{(i+1) !} \prod_{j=0}^{i}(\eta-2 i+j)\right] \bar{\Phi}_{m}^{\eta-2 i-1} \bar{\Phi}_{x}^{i+1}
$$

with $\bar{\Phi}_{m}=\Phi_{m}+\Gamma_{n} \bar{\Theta}, \bar{\Phi}_{x}=\Gamma_{n} \Phi_{x}$ and $\lfloor\cdot\rfloor$ is the floor function. Note that the number of terms on the right hand side of (11) is finite and is equal to $p+1$. Furthermore, from [39], it can be shown that the order of the norm of each term on the right hand side of (11) is at most $O\left(T^{2}\right)$ and that, therefore, $\bar{\delta}_{k}$ is bounded. Finally, since $O\left(T^{2}\right)$ terms are summed $p+1$ times, the order of $\left\|\bar{\delta}_{k+p}\right\|$ is at most $O(p T+T) \cdot O(T)=O(\tau+T) \cdot O(T)$. The control law is then selected as

$$
\mathbf{u}_{k}=-\hat{\Theta}_{\gamma_{1}, k}^{-1}\left(\hat{\Theta}_{x, k} \bar{\chi}_{k}+\hat{\Theta}_{u, k} \overline{\boldsymbol{\xi}}_{k}-\Theta_{r} \mathbf{r}_{k}\right)
$$

where $\hat{\Theta}_{x, k}, \hat{\Theta}_{u, k}, \hat{\Theta}_{\gamma_{1}, k}$ are the estimates of $\Theta_{x}, \Theta_{u}, \Theta_{\gamma_{1}}$ respectively and $\Theta_{r} \in \Re^{m \times q}$ is selected such that $\Gamma_{m}=\Gamma_{n} \Theta_{r}$. In order to derive the adaptive law for $\hat{\Theta}_{x, k}, \hat{\Theta}_{u, k}$ and $\hat{\Theta}_{\gamma_{1}, k}$ it is necessary to derive the closed-loop system.

Consider a $p$ sampling instants delayed (10), adding and subtracting the term $\Gamma_{n} \hat{\Theta}_{\gamma_{1}, k-p} \mathbf{u}_{k-p}$ it is obtained that

$$
\begin{aligned}
\mathbf{x}_{k+1}= & \Phi_{m} \mathbf{x}_{k}+\Gamma_{n}\left(\Theta_{x} \bar{\chi}_{k-p}+\Theta_{u} \bar{\xi}_{k-p}+\Theta_{\gamma_{1}} \mathbf{u}_{k-p}\right. \\
& \left.-\hat{\Theta}_{\gamma_{1}, k-p} \mathbf{u}_{k-p}+\hat{\Theta}_{\gamma_{1}, k-p} \mathbf{u}_{k-p}\right)+\bar{\delta}_{k},
\end{aligned}
$$


Substitution of (13) in (14) and defining the estimation errors as $\tilde{\Theta}_{x, k}=\Theta_{x}-\hat{\Theta}_{x, k}, \tilde{\Theta}_{u, k}=\Theta_{u}-\hat{\Theta}_{u, k}$ and $\tilde{\Theta}_{\gamma_{1}, k}=\Theta_{\gamma_{1}}-\hat{\Theta}_{\gamma_{1}, k}$ the closed-loop error dynamics is obtained as

$$
\begin{aligned}
\mathbf{e}_{k+1}= & \Phi_{m} \mathbf{e}_{k}+\Gamma_{n}\left(\tilde{\Theta}_{x, k-p} \bar{\chi}_{k-p}+\tilde{\Theta}_{u, k-p} \overline{\boldsymbol{\xi}}_{k-p}\right. \\
& \left.+\tilde{\Theta}_{\gamma_{1}, k-p} \mathbf{u}_{k-p}\right)+\bar{\delta}_{k},
\end{aligned}
$$

where $\mathbf{e}_{k}=\mathbf{x}_{k}-\mathbf{x}_{m, k}$. (15) can be rewritten as

$$
\mathbf{e}_{k+1}=\Phi_{m} \mathbf{e}_{k}+\Gamma_{n} \tilde{\Psi}_{k-p}^{\top} \overline{\boldsymbol{\zeta}}_{k-p}+\bar{\delta}_{k}
$$

where $\tilde{\Psi}_{k}^{\top}=\left[\tilde{\Theta}_{x, k}\left|\tilde{\Theta}_{u, k}\right| \tilde{\Theta}_{\gamma_{1}, k}\right] \in \Re^{m \times(2 n+m(p+3))} \quad$ and $\quad \overline{\boldsymbol{\zeta}}_{k}^{\top}=$ $\left[\bar{\chi}_{k}^{\top}\left|\overline{\boldsymbol{\xi}}_{k}^{\top}\right| u_{k}^{\top}\right] \in \Re^{2 n+m(p+3)}$. Defining $\mathbf{z}_{k+1}=D_{\gamma}\left(\mathbf{e}_{k+1}-\Phi_{m} \mathbf{e}_{k}\right)$ and substituting (16) it is obtained that

$$
\mathbf{z}_{k+1}=\tilde{\Psi}_{k-p}^{\top} \overline{\boldsymbol{\zeta}}_{k-p}+\bar{v}_{k},
$$

where $\mathbf{z}_{k} \in \Re^{m}$ and $\bar{v}_{k}=D_{\gamma} \bar{\delta}_{k} \in \Re^{m}$. Minimizing $\mathbf{z}_{k+1}$ makes the tracking error follow the dynamics $\mathbf{e}_{k+1}=\Phi_{m} \mathbf{e}_{k}+\bar{\delta}_{k}$. Therefore, the adaptation law is formulated as follows

$$
\hat{\Psi}_{k+1}=\left\{\begin{array}{cc}
\hat{\Psi}_{k-p}+\frac{\alpha_{k} \beta_{k}}{\varphi_{k}} Q \overline{\boldsymbol{\zeta}}_{k-p} \mathbf{z}_{k+1}^{\top} & \forall k \in[p, \infty) \\
\hat{\Psi}_{0} & \forall k \in[0, p)
\end{array},\right.
$$

where $0<\alpha_{k} \leq 1$ is used to ensure a non-singular $\hat{\Theta}_{\gamma_{1}, k}, Q \in$ $\Re^{(2 n+m(p+3)) \times(2 n+m(p+3))}$ is a positive-definite adaptive gain matrix and $\varphi_{k}=1+\overline{\boldsymbol{\zeta}}_{k-p}^{\top} Q \overline{\boldsymbol{\zeta}}_{k-p}$. Considering that the disturbance term $\mathbf{f}(t)$ is bounded then there exists a bound on $\bar{v}_{k}$ such that the weighing coefficient $\beta_{k}$ defined as

$$
\beta_{k}=\left\{\begin{array}{cl}
1-\frac{\bar{v}_{\max }}{\left\|\mathbf{z}_{k+1}\right\|}, & \text { if }\left\|\mathbf{z}_{k+1}\right\| \geq \bar{v}_{\max } \\
0, & \text { if }\left\|\mathbf{z}_{k+1}\right\|<\bar{v}_{\max }
\end{array},\right.
$$

where $\left\|\bar{v}_{k}\right\| \leq \bar{v}_{\text {max }} \in O(\tau+T) \cdot O(T)$, ensures that the closed-loop system is robust to the influence of the term $\bar{v}_{k}$.

Remark 4: In order for $\hat{\Theta}_{\gamma_{1}, k}$ to be non-singular then, using the approach in [31], $\alpha_{k}^{-1}$ must be selected such that

$$
\alpha_{k}^{-1} \neq \lambda\left[-\hat{\Theta}_{\gamma_{1}, k-p}^{-1} S \frac{\beta_{k}}{\varphi_{k}} Q \overline{\boldsymbol{\zeta}}_{k-p} \mathbf{z}_{k+1}^{\top}\right]
$$

where $\lambda[\cdot]$ is the set of eigenvalues and $S=\left[\begin{array}{llll}\mathbf{0} & \cdots & \mathbf{0} & I\end{array}\right] \in \Re^{m \times(2 n+}$ $m(p+3))$.

\section{B. Stability Analysis}

Theorem 1: The closed loop system, consisting of the plant (3), control input (13) and the adaptive law (18), together with the reference model (4), results in a closed-loop system with a bounded $\tilde{\Psi}_{k}$ and $\lim _{k \rightarrow \infty}\left\|\mathbf{e}_{k}\right\| \leq \epsilon \in O(\tau+T)$.

Proof: To proceed with the proof, let $\mathbf{z}_{k}^{\top}=\left[z_{1, k}\left|z_{2, k}\right| \cdots \mid z_{m, k}\right]^{\top}$ and $\tilde{\Psi}_{k}^{\top}=\left[\tilde{\boldsymbol{\psi}}_{1, k}\left|\tilde{\boldsymbol{\psi}}_{2, k}\right| \cdots \mid \tilde{\boldsymbol{\psi}}_{m, k}\right]^{\top}$, where $\tilde{\boldsymbol{\psi}}_{j, k} \in \Re^{(2 n+m(p+3)) \times 1}$ and $j=1, \ldots, m$. Now, consider the following positive function

$$
V_{k}=\sum_{j=1}^{m}\left[\sum_{i=0}^{p} \tilde{\boldsymbol{\psi}}_{j, k-i}^{\top} Q^{-1} \tilde{\boldsymbol{\psi}}_{j, k-i}\right] \text {. }
$$

The forward difference, $\Delta V_{k}=V_{k+1}-V_{k}$, of (20) is given by

$$
\Delta V_{k}=\sum_{j=1}^{m}\left[\tilde{\boldsymbol{\psi}}_{j, k+1}^{\top} Q^{-1} \tilde{\boldsymbol{\psi}}_{j, k+1}-\tilde{\boldsymbol{\psi}}_{j, k-p}^{\top} Q^{-1} \tilde{\boldsymbol{\psi}}_{j, k-p}\right] .
$$

Consider the adaptive law (18), subtracting both sides from $\boldsymbol{\psi}_{j}$ and defining $\tilde{\boldsymbol{\psi}}_{j, k}=\boldsymbol{\psi}_{j}-\hat{\boldsymbol{\psi}}_{j, k}$ it is obtained that

$$
\tilde{\boldsymbol{\psi}}_{j, k+1}=\tilde{\boldsymbol{\psi}}_{j, k-p}-\frac{\alpha_{k} \beta_{k}}{\varphi_{k}} Q \overline{\boldsymbol{\zeta}}_{k-p} z_{j, k+1}
$$

Substituting (22) in (21) it is obtained that

$$
\Delta V_{k}=\sum_{j=1}^{m}\left[\frac{-2 \alpha_{k} \beta_{k} \tilde{\boldsymbol{\psi}}_{j, k-p}^{\top}}{\varphi_{k}}+\frac{\alpha_{k}^{2} \beta_{k}^{2} \overline{\boldsymbol{\zeta}}_{k-p}^{\top} Q z_{j, k+1}}{\varphi_{k}^{2}}\right] \overline{\boldsymbol{\zeta}}_{k-p} z_{j, k+1}
$$

Using the fact that $\frac{\alpha_{k} \overline{\boldsymbol{\zeta}}_{k-p}^{\top} Q \overline{\boldsymbol{\zeta}}_{k-p}}{\varphi_{k}}<1,(23)$ is reduced as

$$
\begin{aligned}
\Delta V_{k} & \leq \sum_{j=1}^{m}\left[\frac{-2 \alpha_{k} \beta_{k} \tilde{\boldsymbol{\psi}}_{j, k-p}^{\top} \overline{\boldsymbol{\zeta}}_{k-p} z_{j, k+1}}{\varphi_{k}}+\frac{\alpha_{k} \beta_{k}^{2} z_{j, k+1}^{2}}{\varphi_{k}}\right] \\
& \leq-\frac{2 \alpha_{k} \beta_{k}}{\varphi_{k}} \overline{\boldsymbol{\zeta}}_{k-p}^{\top} \tilde{\Psi}_{k-p} \mathbf{z}_{k+1}+\frac{\alpha_{k} \beta_{k}^{2}}{\varphi_{k}} \mathbf{z}_{k+1}^{\top} \mathbf{z}_{k+1} .
\end{aligned}
$$

Furthermore, from (19) it is obtained that

$$
\beta_{k}^{2}\left\|\mathbf{z}_{k+1}\right\|^{2}=\beta_{k}\left\|\mathbf{z}_{k+1}\right\|^{2}-\beta_{k} \bar{v}_{\max } \cdot\left\|\mathbf{z}_{k+1}\right\| .
$$

Thus, substituting (17) and (25) in (24), it is obtained that

$$
\Delta V_{k} \leq-\frac{\alpha_{k} \beta_{k}^{2}}{\varphi_{k}} \mathbf{z}_{k+1}^{\top} \mathbf{z}_{k+1}
$$

which implies that $V_{k}$ is non-increasing and, thus, $\tilde{\Psi}_{k}$ is bounded. Note that for any $k \in\left[k_{0}, \infty\right)$ the following is true

$$
V_{k+1}=V_{k_{0}}+\sum_{i=0}^{k-k_{0}} \Delta V_{k_{0}+i}
$$

Substituting (26) in (27) it is obtained that

$$
\lim _{k \rightarrow \infty} V_{k+1} \leq \max _{k_{0} \in[0, p)} V_{k_{0}}-\lim _{k \rightarrow \infty} \sum_{i=0}^{k-k_{0}} \frac{\alpha_{k} \beta_{k}^{2}}{\varphi_{k_{0}+i}} \mathbf{z}_{k_{0}+i+1}^{\top} \mathbf{z}_{k_{0}+i+1} .
$$

Consider that $V_{k+1}$ is non-negative and $V_{k_{0}}$ is finite in the interval $[0, p)$, then it is obtained that

$$
\lim _{k \rightarrow \infty} \frac{\alpha_{k} \beta_{k}^{2}}{\varphi_{k}} \mathbf{z}_{k+1}^{\top} \mathbf{z}_{k+1}=0 .
$$

To guarantee that $\lim _{k \rightarrow \infty} \beta_{k}\left\|\mathbf{z}_{k+1}\right\|=0$ it must be guaranteed that $\left\|\overline{\boldsymbol{\zeta}}_{k}\right\| \leq \mu_{0}+\mu_{1} \max _{i \in[0, k+1]}\left\|\mathbf{z}_{i}\right\|$. Consider the relationship between $\mathbf{e}_{k}$ and $\mathbf{z}_{k}$ given as $\mathbf{e}_{k+1}=\Phi_{m} \mathbf{e}_{k}+\Gamma_{n} \mathbf{z}_{k+1}+\left[I-\Gamma_{n} D_{\gamma}\right] \bar{\delta}_{k}$. Using the fact that $\Phi_{m}$ has eigenvalues inside the unit-circle, $\mathbf{e}_{k}=$ $\mathbf{x}_{m, k}-\mathbf{x}_{k}$ and that $\mathbf{x}_{m, k}$ is bounded then there exists constants $c_{0}$ and $c_{1}$ such that, [32],

$$
\left\|\mathbf{x}_{k+1}\right\| \leq c_{0}+c_{1}\left\|\mathbf{z}_{k+1}\right\|,
$$

and, from Assumption 1, the control input is bounded as

$$
\left\|\mathbf{u}_{k-p}\right\| \leq \kappa_{0}+\kappa_{1} \max _{i \in[0, k+1]}\left\|\mathbf{x}_{i}\right\|,
$$

for some constant $\kappa_{0}$ and $\kappa_{1}$. Looking at the signal growth rates, $\overline{\boldsymbol{\zeta}}_{k}$ is a vector containing $\mathbf{x}_{k}$ and $\mathbf{u}_{k}$, then there exists

$$
\left\|\overline{\boldsymbol{\zeta}}_{k-p}\right\| \leq c_{0}^{0}+c_{1}^{0}\left\|\mathbf{z}_{k}\right\| \leq c_{0}^{0}+c_{1}^{0} \max _{i \in[0, k+1]}\left\|\mathbf{z}_{i}\right\|
$$

and, therefore, using the Key Technical Lemma, [31], [32], $\lim _{k \rightarrow \infty} \beta_{k}\left\|\mathbf{z}_{k+1}\right\|=0$. Further, using the definition of $\beta_{k}$ given by (25), $z_{k+1}$ will converge to a bound of $\left|\bar{v}_{k}\right| \in O(\tau+T) \cdot O(T)$ and from the definition of $\mathbf{z}_{k}$, as $k \rightarrow \infty$, the following stable error dynamics is achieved

$$
\mathbf{e}_{k+1}=\Phi_{m} \mathbf{e}_{k}+\bar{v}_{k}
$$

and, based on [37], $\lim _{k \rightarrow \infty}\left\|\mathbf{e}_{k}\right\| \leq \epsilon \in O(\tau+T)$ is guaranteed. 


\section{EXtension to the Uncertain Time-Delay CASE}

Consider the system (1) with an uncertain input time-delay $\tau$ such that its sampled-data representation is given as

$$
\mathbf{x}_{k+1}=\Phi \mathbf{x}_{k}+\Gamma_{1} \mathbf{u}_{k-\ell}+\Gamma_{2} \mathbf{u}_{k-\ell-1}+\mathbf{d}_{k}
$$

where the delay $\ell \in Z$ is uncertain and given as $\ell T<\tau<(\ell+1) T$. The delay is assumed to have an upper-bound as $\tau \leq \tau_{p}$, where $\tau_{p}$ is an integer multiple of the sampling-interval, i.e. $\tau_{p}=p T$ for some known $p$. Similar to the known time-delay problem, the controller design is preceded by the reformulation of the system dynamics (34) such that the influence of the disturbance $\mathbf{d}_{k}$ is minimized. This is accomplished by using the delay observer (7) which in this case is given by

$$
\mathbf{w}_{k-1}=D_{\gamma}\left(\mathbf{x}_{k}-\Phi \mathbf{x}_{k-1}\right)-\Theta_{\gamma_{1}} \mathbf{u}_{k-\ell-1}-\Theta_{\gamma_{2}} \mathbf{u}_{k-\ell-2}+O\left(T^{3}\right)
$$

Substituting (5) and (35) in (34) it is obtained that

$$
\begin{aligned}
\mathbf{x}_{k+1}= & \Phi_{m} \mathbf{x}_{k}+\Gamma_{n}\left(\bar{\Theta} \mathbf{x}_{k}-\Phi_{x} \mathbf{x}_{k-1}+\Theta_{\gamma_{1}} \mathbf{u}_{k-\ell}+\Theta_{\gamma_{21}} \mathbf{u}_{k-\ell-1}\right. \\
& \left.-\Theta_{\gamma_{2}} \mathbf{u}_{k-\ell-2}\right)+\bar{\varsigma}_{k}
\end{aligned}
$$

Performing successive substitutions on (36), it is obtained that

$$
\begin{aligned}
\mathbf{x}_{k+p+1}= & \Phi_{m} \mathbf{x}_{k+p}+\Gamma_{n}\left(\Theta_{x} \bar{\chi}_{k}+\Theta_{u} \bar{\xi}_{k+p-\ell}+\Theta_{\gamma_{1}} \mathbf{u}_{k+p-\ell}\right) \\
& +\bar{\delta}_{k+p},
\end{aligned}
$$

where $\Theta_{x}, \Theta_{u}$ are defined similar to in (10) and $\overline{\boldsymbol{\xi}}_{k+p-\ell}^{\top}=\left[\mathbf{u}_{k+p-\ell-1}^{\top}\right.$ $\left.|\cdots| \mathbf{u}_{k-\ell-2}^{\top}\right] \in \Re^{m(p+2)}$. Let $\Theta_{u}=\left[\Omega_{\ell+1}|\cdots| \Omega_{\ell+p+2}\right] \in \Re^{m \times m}$ $(p+2)$ and revise (37) such that

$$
\begin{aligned}
\mathbf{x}_{k+p+1}= & \Phi_{m} \mathbf{x}_{k+p}+\Gamma_{n}\left(\Theta_{x} \bar{\chi}_{k}+\Omega_{\ell} \mathbf{u}_{k+p-\ell}+\Omega_{\ell+1} \mathbf{u}_{k+p-\ell-1}\right. \\
& \left.+\cdots+\Omega_{p} \mathbf{u}_{k}+\cdots+\Omega_{\ell+p+2} \mathbf{u}_{k-\ell-2}\right)+\bar{\delta}_{k+p},
\end{aligned}
$$

where $\Omega_{\ell}=\Theta_{\gamma_{1}}$ for convenience. Let the matrices $\Omega_{1}, \ldots, \Omega_{\ell-1}$ and $\Omega_{\ell+p+3}, \ldots, \Omega_{2 p+2}$ be null matrices such that (38) is written as

$$
\begin{aligned}
\mathbf{x}_{k+p+1}= & \Phi_{m} \mathbf{x}_{k+p}+\Gamma_{n}\left(\Theta_{x} \bar{\chi}_{k}+\Omega_{1} \mathbf{u}_{k+p-1}+\cdots\right. \\
& +\Omega_{\ell-1} \mathbf{u}_{k+p-\ell+1}+\Omega_{\ell} \mathbf{u}_{k+p-\ell}+\Omega_{\ell+1} \mathbf{u}_{k+p-\ell-1}+\ldots \\
& +\Omega_{p} \mathbf{u}_{k}+\cdots+\Omega_{\ell+p+2} \mathbf{u}_{k-\ell-2}+\Omega_{\ell+p+3} \mathbf{u}_{k-\ell-3}+\cdots \\
& \left.+\Omega_{2 p+2} \mathbf{u}_{k-p-2}\right)+\bar{\delta}_{k+p} .
\end{aligned}
$$

Now define $\bar{\Omega}_{1}=\left[\Omega_{1}|\cdots| \Omega_{p-1}\right] \in \Re^{m \times m(p-1)}$ and $\bar{\Omega}_{2}=\left[\Omega_{p+1} \mid\right.$ $\left.\cdots \mid \Omega_{2 p+2}\right] \in \Re^{m \times m(p+2)}$ such that (40) simplifies as

$$
\begin{aligned}
\mathbf{x}_{k+p+1}= & \Phi_{m} \mathbf{x}_{k+p}+\Gamma_{n}\left(\Theta_{x} \bar{\chi}_{k}+\bar{\Omega}_{1} \overline{\mathbf{u}}_{k+p}+\Omega_{p} \mathbf{u}_{k}+\bar{\Omega}_{2} \bar{\xi}_{k}\right) \\
& +\bar{\delta}_{k+p},
\end{aligned}
$$

where $\overline{\mathbf{u}}_{k}^{\top}=\left[\mathbf{u}_{k-1}^{\top}|\cdots| \mathbf{u}_{k-p+1}^{\top}\right] \in \Re^{m(p-1)}$. The control law can then be selected as

$$
\mathbf{u}_{k}=-\hat{\Omega}_{p, k}^{-1}\left(\hat{\Theta}_{x, k} \bar{\chi}_{k}+\hat{\bar{\Omega}}_{2, k} \bar{\xi}_{k}-\Theta_{r} \mathbf{r}_{k}\right) .
$$

where $\hat{\Omega}_{p, k}, \hat{\Theta}_{x, k}$ and $\hat{\bar{\Omega}}_{2, k}$ are the estimates of $\Omega_{p}, \Theta_{x}$ and $\bar{\Omega}_{2}$ respectively.

To select that adaptive law for the parameters $\hat{\Omega}_{p, k}, \hat{\Theta}_{x, k}$ and $\hat{\bar{\Omega}}_{2, k}$, substitute (41) in a $p$ sampling instants delayed (40) and subtract the result from the reference model (4) such that the error dynamics is obtained as

$$
\mathbf{e}_{k+1}=\Phi_{m} \mathbf{e}_{k}+\Gamma_{n} \tilde{\Psi}_{k-p}^{\top} \overline{\boldsymbol{\zeta}}_{k-p}+\Gamma_{n} \bar{\Omega}_{1}^{\top} \overline{\mathbf{u}}_{k}+\bar{\delta}_{k} .
$$

where $\tilde{\Psi}_{k}^{\top}=\left[\tilde{\Theta}_{x, k}\left|\tilde{\bar{\Omega}}_{2, k}\right| \tilde{\Omega}_{p, k}\right] \in \Re^{m \times(2 n+m(p+3))}$ and $\overline{\boldsymbol{\zeta}}_{k}^{\top}=\left[\bar{\chi}_{k}^{\top} \mid\right.$ $\left.\overline{\boldsymbol{\xi}}_{k}^{\top} \mid \mathbf{u}_{k}^{\top}\right] \in \Re^{2 n+m(p+3)}$. Note that the error dynamics (42) is similar to (16) with the only difference being the additional term $\Gamma_{n} \bar{\Omega}_{1}^{\top} \overline{\mathbf{u}}_{k}$ which exists due to the uncertainty in the delay. If $\ell$ is known and $\ell=p$ then $\bar{\Omega}_{1}$ would be a null matrix.

Remark 5: The number of terms in $\bar{\Omega}_{1} \overline{\mathbf{u}}_{k+p}$ increases with increasing delay upper-bound, p, and decreasing the sampling-interval, $\mathrm{T}$, values. An increase in the number of terms in $\bar{\Omega}_{1} \overline{\mathbf{u}}_{k+p}$ may degrade the performance and therefore, while it is desirable to use smaller $\mathrm{T}$ in order to reduce the effect of the disturbances, care must be taken to pick a suitable value.

Using $\mathbf{z}_{k+1}=D_{\gamma}\left(\mathbf{e}_{k+1}-\Phi_{m} \mathbf{e}_{k}\right)$ it is obtained that

$$
\mathbf{z}_{k+1}=\tilde{\Psi}_{k-p}^{\top} \overline{\boldsymbol{\zeta}}_{k-p}+\bar{\Omega}_{1}^{\top} \overline{\mathbf{u}}_{k}+\bar{v}_{k},
$$

where $\mathbf{z}_{k+1} \in \Re^{m}$. Based on (43), and following an approach similar to that in [41] the adaptation law is proposed as

$$
\hat{\Psi}_{k+1}=\left\{\begin{array}{cl}
\hat{\Psi}_{k-p}+\alpha_{k} \frac{\beta_{k}}{\vartheta_{k}} Q \overline{\boldsymbol{\zeta}}_{k-p} \mathbf{z}_{k+1}^{\top} & \forall k \in[p, \infty) \\
\hat{\Psi}_{0} & \forall k \in[0, p)
\end{array}\right.
$$

where the scalar function $\vartheta_{k}=1+\alpha_{k} \overline{\boldsymbol{\zeta}}_{k-p}^{\top} Q \overline{\boldsymbol{\zeta}}_{k-p}+\alpha_{k} \gamma_{c} \lambda_{c}^{2}\left\|\overline{\mathbf{u}}_{k}\right\|^{2}$, the matrix $Q$ is a constant positive definite adaptive gain matrix of dimension $2 n+m(p+3), \gamma_{c}, \lambda_{c}$ are positive tuning constants, $\beta_{k}$ is a positive weighing coefficient and $\alpha_{k}>0$ is a coefficient used to ensure a nonsingular $\hat{\Omega}_{p, k}$.

Remark 6: $\alpha_{k}$ must be selected such that $\alpha_{k}^{-1}$ is not an eigenvalue of $-\hat{\Omega}_{p, k-p}^{-1} S \frac{\beta_{k}}{\vartheta_{k}} Q \overline{\boldsymbol{\zeta}}_{k-p} \mathbf{z}_{k+1}^{\top}$ where $S=\left[\begin{array}{lll}\mathbf{0} & \cdots & \mathbf{0}\end{array}\right] \in$ $\Re^{m \times(n+m(p+3))}$.

Assuming that $\left\|\bar{\Omega}_{1}\right\|=\lambda_{c} \rho$, where $\rho$ is an uncertain positive constant, it is seen that $\left\|\bar{\Omega}_{1}^{\top} \overline{\mathbf{u}}_{k}\right\| \leq \lambda_{c} \rho\left\|\overline{\mathbf{u}}_{k}\right\|$. The weighing coefficient $\beta_{k}$ is defined as,

$\beta_{k}= \begin{cases}1-\frac{\lambda_{c} \hat{\rho}_{k}\left\|\overline{\mathbf{u}}_{k}\right\|+\bar{v}_{\max }}{\left\|\mathbf{z}_{k+1}\right\|}, & \text { if }\left\|\mathbf{z}_{k+1}\right\| \geq \lambda_{c} \hat{\rho}_{k}\left\|\overline{\mathbf{u}}_{k}\right\|+\bar{v}_{\max } \\ 0, & \text { if }\left\|\mathbf{z}_{k+1}\right\|<\lambda_{c} \hat{\rho}_{k}\left\|\overline{\mathbf{u}}_{k}\right\|+\bar{v}_{\max }\end{cases}$

where $\left\|\bar{v}_{k}\right\| \leq \bar{v}_{\max }, \hat{\rho}_{k}$ is the estimate of $\rho$ and $\lambda_{c}$ is chosen as any constant as long as it satisfies $0<\lambda_{c}<\lambda_{c, \max }$, with $\lambda_{\mathrm{c}, \max }$ being defined later. The adaptive law for $\rho$ is given as

$$
\hat{\rho}_{k+1}=\hat{\rho}_{k}+\alpha_{k} \frac{\beta_{k} \lambda_{c} \gamma_{c}\left\|\overline{\mathbf{u}}_{k}\right\| \cdot\left\|\mathbf{z}_{k+1}\right\|}{\vartheta_{k}} .
$$

Using (45), it is obtained that

$$
\begin{aligned}
\beta_{k}^{2} \mathbf{z}_{k+1}^{\top} \mathbf{z}_{k+1}= & \beta_{k} \mathbf{z}_{k+1}^{\top} \mathbf{z}_{k+1}-\beta_{k}\left(\lambda_{c} \hat{\rho}_{k}\left\|\overline{\mathbf{u}}_{k}\right\|+\bar{v}_{\max }\right) \\
& \cdot\left\|\mathbf{z}_{k+1}\right\| .
\end{aligned}
$$

Theorem 2: Under the adaptation law (44) and the closed-loop dynamics (43) the tracking error $\mathbf{e}_{k}$ is bounded if $\lambda_{c} \bar{\rho} c_{2}^{1}<1$, where $\bar{\rho}$ is an upper bound on $\hat{\rho}_{k}$ and $c_{2}^{1}$ is a constant obtained from the bound on $\bar{\zeta}_{k}$.

Proof: Consider the positive function given as

$$
V_{k}=\sum_{j=1}^{m}\left(\sum_{i=k-p}^{k} \tilde{\boldsymbol{\psi}}_{j, i}^{\top} Q^{-1} \tilde{\boldsymbol{\psi}}_{j, i}\right)+\frac{1}{\gamma_{c}} \tilde{\rho}_{k}^{2}
$$


where $\tilde{\Psi}_{k}^{\top}=\left[\tilde{\boldsymbol{\psi}}_{1, k}\left|\tilde{\boldsymbol{\psi}}_{2, k}\right| \cdots \mid \tilde{\boldsymbol{\psi}}_{m, k}\right]^{\top}$. The forward difference, $\Delta V_{k}=$ $V_{k+1}-V_{k}$, is given as

$$
\begin{aligned}
\Delta V_{k}= & \sum_{j=1}^{m}\left(\tilde{\boldsymbol{\psi}}_{j, k+1}^{\top} Q^{-1} \tilde{\boldsymbol{\psi}}_{j, k+1}-\tilde{\boldsymbol{\psi}}_{j, k-p}^{\top} Q^{-1} \tilde{\boldsymbol{\psi}}_{j, k-p}\right) \\
& +\frac{1}{\gamma_{c}}\left(\tilde{\rho}_{k+1}^{2}-\tilde{\rho}_{k}^{2}\right) .
\end{aligned}
$$

Defining $\tilde{\rho}_{k}=\rho-\hat{\rho}_{k}$, then it is possible to obtain

$$
\tilde{\rho}_{k+1}=\tilde{\rho}_{k}-\alpha_{k} \frac{\beta_{k} \lambda_{c} \gamma_{c}\left\|\overline{\mathbf{u}}_{k}\right\| \cdot\left\|\mathbf{z}_{k+1}\right\|}{\vartheta_{k}} .
$$

Substituting (44) and (50) in (49) it is obtained that

$$
\begin{aligned}
\Delta V_{k}= & \sum_{j=1}^{m}\left(\tilde{\boldsymbol{\psi}}_{j, k-p}-\alpha_{k} \frac{\beta_{k}}{\vartheta_{k}} Q \overline{\boldsymbol{\zeta}}_{k-p} z_{j, k+1}\right)^{\top} Q^{-1}\left(\tilde{\boldsymbol{\psi}}_{j, k-p}\right. \\
& \left.-\alpha_{k} \frac{\beta_{k}}{\vartheta_{k}} Q \overline{\boldsymbol{\zeta}}_{k-p} z_{j, k+1}\right)-\sum_{j=1}^{m} \tilde{\boldsymbol{\psi}}_{j, k-p}^{\top} Q^{-1} \tilde{\boldsymbol{\psi}}_{j, k-p} \\
& +\frac{1}{\gamma_{c}}\left(\tilde{\rho}_{k}-\frac{\alpha_{k} \beta_{k} \lambda_{c} \gamma_{c}\left\|\overline{\mathbf{u}}_{k}\right\|\left\|\mathbf{z}_{k+1}\right\|}{\vartheta_{k}}\right)^{2}-\frac{1}{\gamma_{c}} \tilde{\rho}_{k}^{2} \\
\leq & \sum_{j=1}^{m}\left(\frac{\alpha_{k}^{2} \beta_{k}^{2}}{\vartheta_{k}^{2}} \overline{\boldsymbol{\zeta}}_{k-p}^{\top} Q z_{j, k+1}-2 \frac{\alpha_{k} \beta_{k}}{\vartheta_{k}} \tilde{\boldsymbol{\psi}}_{j, k-p}^{\top}\right) \overline{\boldsymbol{\zeta}}_{k-p} z_{j, k+1} \\
& +\frac{\alpha_{k}^{2} \beta_{k}^{2}}{\vartheta_{k}^{2}} \gamma_{c} \lambda_{c}^{2}\left\|\overline{\mathbf{u}}_{k}\right\|^{2} \mathbf{z}_{k+1}^{\top} \mathbf{z}_{k+1}-2 \frac{\alpha_{k} \beta_{k}}{\vartheta_{k}} \lambda_{c} \tilde{\rho}_{k}\left\|\overline{\mathbf{u}}_{k}\right\|\left\|\mathbf{z}_{k+1}\right\| \\
\leq & -2 \frac{\alpha_{k} \beta_{k}}{\vartheta_{k}} \mathbf{z}_{k+1}^{\top} \tilde{\Psi}_{k-p}^{\top} \overline{\boldsymbol{\zeta}}_{k-p}-2 \frac{\alpha_{k} \beta_{k}}{\vartheta_{k}} \lambda_{c} \tilde{\rho}_{k}\left\|\overline{\mathbf{u}}_{k}\right\|\left\|\mathbf{z}_{k+1}\right\| \\
& +\frac{\alpha_{k}^{2} \beta_{k}^{2}}{\vartheta_{k}^{2}}\left(\overline{\boldsymbol{\zeta}}_{k-p}^{\top} Q \overline{\boldsymbol{\zeta}}_{k-p}+\gamma_{c} \lambda_{c}^{2}\left\|\overline{\mathbf{u}}_{k}\right\|^{2}\right) \mathbf{z}_{k+1}^{\top} \mathbf{z}_{k+1} .
\end{aligned}
$$

Consider the first term on the right hand side of (51), using the definition of $\mathbf{z}_{k+1}$ in (43) and (47) it is obtained that

$$
\begin{aligned}
-2 \frac{\alpha_{k} \beta_{k}}{\vartheta_{k}} \mathbf{z}_{k+1}^{\top} \tilde{\Psi}_{k-p}^{\top} \overline{\boldsymbol{\zeta}}_{k-p}= & -2 \frac{\alpha_{k} \beta_{k}}{\vartheta_{k}} \mathbf{z}_{k+1}^{\top}\left[\mathbf{z}_{k+1}-\bar{\Omega}_{1}^{\top} \overline{\mathbf{u}}_{k}-\bar{v}_{k}\right] \\
\leq & -2 \frac{\alpha_{k} \beta_{k}}{\vartheta_{k}}\left[\mathbf{z}_{k+1}^{\top} \mathbf{z}_{k+1}-\left(\lambda_{c} \rho\left\|\overline{\mathbf{u}}_{k}\right\|\right.\right. \\
& \left.\left.+\bar{v}_{\max }\right)\left\|\mathbf{z}_{k+1}\right\|\right]
\end{aligned}
$$

Substituting (52) in (51) and simplifying, it is obtained that

$$
\Delta V_{k} \leq-\frac{\alpha_{k} \beta_{k}^{2}}{\vartheta_{k}}\left[2-\frac{\alpha_{k}}{\vartheta_{k}}\left(\overline{\boldsymbol{\zeta}}_{k-p}^{\top} Q \overline{\boldsymbol{\zeta}}_{k-p}+\gamma_{c} \lambda_{c}^{2}\left\|\overline{\mathbf{u}}_{k}\right\|^{2}\right)\right] \mathbf{z}_{k+1}^{\top} \mathbf{z}_{k+1}
$$

Using $\frac{\alpha_{k} \overline{\boldsymbol{\zeta}}_{k-p}^{\top} Q \overline{\boldsymbol{\zeta}}_{k-p}+\alpha_{k} \gamma_{c} \lambda_{c}^{2}\left\|\overline{\mathbf{u}}_{k}\right\|^{2}}{\vartheta_{k}}<1$ it is obtained that

$$
\Delta V_{k}<-\alpha_{k} \frac{\beta_{k}^{2}}{\vartheta_{k}} \mathbf{z}_{k+1}^{\top} \mathbf{z}_{k+1} .
$$

Following the same steps in Theorem 1, it is concluded that

$$
\lim _{k \rightarrow \infty} \alpha_{k} \frac{\beta_{k}^{2}}{\vartheta_{k}} \mathbf{z}_{k+1}^{\top} \mathbf{z}_{k+1}=0 .
$$

The result (54) shows that $\tilde{\Theta}_{x, k}, \tilde{\Omega}_{p, k}, \tilde{\Omega}_{2, k}$ and $\tilde{\rho}_{k}$ are bounded. Using arguments similar to in Theorem 1 , it can be obtained that $\left\|\overline{\boldsymbol{\zeta}}_{k}\right\| \leq c_{1}^{1}+c_{2}^{1}\left\|\mathbf{z}_{k+1}\right\|$ for positive constants $c_{1}^{1}$ and $c_{2}^{1}$, satisfying the condition required by the Key Technical Lemma that guarantees that $\lim _{k \rightarrow \infty} \beta_{k}\left\|\mathbf{z}_{k+1}\right\|=0$. There exists a positive constant $\varepsilon$ such

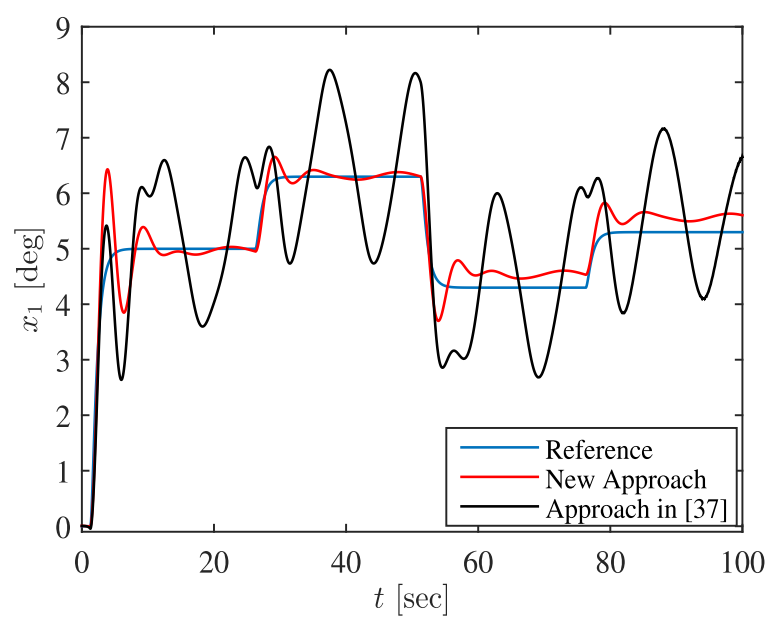

Fig. 1. Performance of the proposed approach vs the approach in [37].

that $\max _{i \in[0, k]}\left\{\beta_{i}\left\|\mathbf{z}_{i+1}\right\|\right\} \leq \varepsilon$. Then according to the definition of $\beta_{k}$ in (45)

$$
\max _{i \in[0, k]}\left\|\mathbf{z}_{i+1}\right\| \leq \varepsilon+\lambda_{c} \bar{\rho} \max _{i \in[0, k]}\left\|\overline{\mathbf{u}}_{i}\right\| \leq \varepsilon+\lambda_{c} \bar{\rho} \max _{i \in[0, k]}\left\|\overline{\boldsymbol{\zeta}}_{i}\right\|
$$

where $\max _{i \in[0, k]} \hat{\rho}_{i} \leq \bar{\rho}$. Following the analysis in Theorem 1 and the bound on $\overline{\boldsymbol{\zeta}}_{k}$ the maximum bound of $\mathbf{z}_{k}$ is found as

$$
\max _{i \in[0, k]}\left\|\mathbf{z}_{i+1}\right\| \leq \varepsilon+\lambda_{c} \bar{\rho} \max _{i \in[0, k]}\left\{c_{1}^{1}+c_{2}^{1}\left\|\mathbf{z}_{i}\right\|\right\}
$$

which results in

$$
\left\|\mathbf{z}_{k+k_{0}}\right\| \leq\left(\lambda_{c} \bar{\rho} c_{2}^{1}\right)^{k}\left\|\mathbf{z}_{k_{0}}\right\|+\left(\varepsilon+\lambda_{c} \bar{\rho} c_{1}^{1}\right) \sum_{i=0}^{k-1}\left(\lambda_{c} \bar{\rho} c_{2}^{1}\right)^{i}
$$

implying that $\left\|\mathbf{z}_{k}\right\|$ is bounded if $\left|\lambda_{c}\right|<\lambda_{\mathrm{c}, \max }<\frac{1}{\bar{\rho} c_{2}^{1}}$.

From (58), the steady-state bound on $\left\|\mathbf{z}_{k}\right\|$ is given as $\left(\varepsilon+\lambda_{c} \bar{\rho}\right.$ $\left.c_{1}^{1}\right) \sum_{i=0}^{k-1}\left(\lambda_{c} \bar{\rho} c_{2}^{1}\right)^{i}$ and, since, $\mathbf{e}_{k+1}=\Phi_{m} \mathbf{e}_{k}+\Gamma_{n} \mathbf{z}_{k+1}+[I-$ $\left.\Gamma_{n} D_{\gamma}\right] \overline{\bar{\delta}}_{k}$ then the bound on $\left\|\mathbf{e}_{k}\right\|$ can be similarly evaluated.

Remark 7: The requirement $\lambda_{c} \bar{\rho} c_{2}^{1}<1$ can be satisfied with a careful tuning of $\lambda_{c}$, or, with a careful selection of the sampling interval $T$ since it effects $\bar{\rho}$. However, since $c_{2}^{1}$ and $\bar{\rho}$ depend on uncertain system dynamics and therefore may not be known a priori, this requirement is an inherent restriction of system structure.

\section{Simulation Example}

Consider the nominal longitudinal dynamics of a four-engine jet aircraft, [42].

$\left[\begin{array}{c}\dot{x}_{1} \\ \dot{x}_{2}\end{array}\right]=\left[\begin{array}{cc}-0.323 & 1 \\ -1.169 & -0.480\end{array}\right]\left[\begin{array}{l}x_{1} \\ x_{2}\end{array}\right]+\left[\begin{array}{c}-0.018 \\ -1.379\end{array}\right](u(t-\tau)+f(t))$

where $x_{1}, x_{2}$ and $u$ are the angle of attack, the pitch rate and the elevator deflection, $f(t)=0.025\left(1+\sin \left(0.5 t-\frac{\pi}{2}\right)\right)$ and $\tau=0.41$ $\mathrm{s}$. To introduce uncertainty, the delay value is assumed to be $0.5 \mathrm{~s}$ during the controller development, elevator effectiveness is decreased by $30 \%$, proximity of the open loop poles to the imaginary axis was halved and the damping ratio was reduced by $48 \%$. To obtain the reference model, the nominal system (59) is stabilized using the LQR method and the closed loop system is sampled at $T=0.02 \mathrm{~s}$ resulting 
in:

$$
\left[\begin{array}{l}
x_{1, k+1}^{m} \\
x_{2, k+1}^{m}
\end{array}\right]=\left[\begin{array}{cc}
0.9924 & 0.0179 \\
-0.0622 & 0.9078
\end{array}\right]\left[\begin{array}{l}
x_{1, k}^{m} \\
x_{2, k}^{m}
\end{array}\right]+\left[\begin{array}{l}
0.0021 \\
0.0905
\end{array}\right] r_{k-p}
$$

The controller parameters are tuned as $\gamma_{c}=50, \lambda_{c}=0.015$ and $Q=$ $\operatorname{diag}(3,0.5,4,1, \ldots, 1)$ while the initial value for $\hat{\rho}_{k}$ is selected as 0.1 . As seen in Fig. 1, the proposed method provides convergence within a reasonable error bound around the desired trajectory as opposed to the approach in [37].

\section{CONCLUSION}

In this work, a discrete version of the Adaptive Posicast Controller is developed for sampled-data systems, in the presence of disturbances and input time-delays that are possibly non-integer multiples of the sampling interval. A disturbance observer is introduced to the controller design, the utilization of which minimizes the effect of the disturbance on the closed loop system performance. In addition, the extension of the method for the case of uncertain input delays is presented.

\section{REFERENCES}

[1] Y. Yildiz, A. Annaswamy, I. Kolmanovsky, and D. Yanakiev, "Adaptive posicast controller for time-delay systems with relative degree $n^{*} \leq 2$," Automatica, vol. 46, pp. 279-289, 2010.

[2] Y. Yildiz, A. Annaswamy, D. Yanakiev, and I. Kolmanovsky, "Spark ignition engine idle speed control: An adaptive control approach," IEEE Trans. Ctrl. Sys. Tech., vol. 19, no. 5, pp. 990-1002, 2011.

[3] Y. Yildiz, A. Annaswamy, D. Yanakiev, and I. Kolmanovsky, "Spark ignition engine fuel-to-air ratio control: An adaptive control approach,' Ctrl. Eng. Prac., vol. 18, no. 12, pp. 1369-1378, 2010.

[4] Z. T. Dydek, A. M. Annaswamy, J. E. Slotine, and E. Lavretsky, "High performance adaptive control in the presence of time-delays," In Proc. Amer. Ctrl. Conf., pp. 880-885, Baltimore, MD, 2010.

[5] O. Smith, "A controller to overcome dead time," ISA J., vol. 6, 1959.

[6] S. I. Niculescu, Delay Effects on Stability: A Robust Control Approach, Springer-Verlag, Heidelberg, Germany, 2001.

[7] S. Majhi and D. Atherton, "A new smith predictor and controller for unstable and integrating processes with time-delay," In Proc. Conf. Dec. Ctrl., Tampa, FL, 1998.

[8] Z. J. Palmor, "Time-delay compensation-smith predictor and its modifications," The Control Handbook, pp. 224-237, edited by W. Levine, CRSC Press, Boca Raton, FL, USA, 1996.

[9] K. J. Astrom, C. C. Hang, and B. C. Lim, "A new smith predictor for controlling a process with an integrator and long dead-time," IEEE Trans. Autom. Ctrl., vol. 39, no. 2, pp. 343-345, 1994.

[10] J.-P. Richard, "Time-delay systems: An overview of some recent advances and open problems," Automatica, vol. 39, pp. 1667-1694, 2003.

[11] K. Gu and S.-I. Niculescu, "Survey on recent results in the stability and control of time-delay systems," J. Dyn. Sys. Meas. Ctrl., vol. 125, no. 2, pp. 158-165, 2003.

[12] Q.-G. Wang, T. H. Lee, and K. K. Tan, Finite Spectrum Assignment for Time-Delay Systems, Vol. 239 of the Lecture Notes in Ctrl. Info. Sc., Springer-Verlag, New York, 1999.

[13] S. Mondie and W. Michiels, "Finite spectrum assignment of unstable time-delay systems with a safe implementation," IEEE Trans. Autom. Ctrl., vol. 48, no. 12, pp. 2207-2212, 2003.

[14] A. Z. Manitius and A. W. Olbrot, "Finite spectrum assignment problem for systems with delays," IEEE Trans. Autom. Ctrl., vol. 24, no. 4, pp. 541553,1979

[15] S.-I. Niculescu and A. M. Annaswamy, "An adaptive smith-controller for time-delay systems with relative degree $n^{*} \leq 2$," Syst. Ctrl. Lett., vol. 49, pp. 347-358, 2003.

[16] R. Ortega and R. Lozano, "Globally stable adaptive controller for system with delay," Int. J. Ctrl., vol. 47, no. 1, pp. 17-23, 1988.
[17] F. Mazenc and S.-I. Niculescu, "Generating positive and stable solutions through delayed state feedback," Automatica, vol. 47, no. 3, pp. 525-533, 2011.

[18] F. Mazenc, S.-I. Niculescu, and M. Bekaik, "Backstepping for nonlinear systems with delay in the input revisited," SIAM J. Ctrl. Optim., vol. 49 pp. 2263-2278, 2011.

[19] F. Mazenc, M. Malisoff, and Z. Lin, "Further results on input-to-state stability for nonlinear systems with delayed feedbacks," Automatica, vol. 44, no. 9, pp. 2415-2421, 2008.

[20] M. Jankovic, "Cross-term forwarding for systems with time-delay," IEEE Trans. Autom. Ctrl., vol. 54, no. 3, pp. 498-511, 2009.

[21] P. Pepe and Z.-P. Jiang, "A Lyapunov-Krasovskii methodology for ISS and iISS of time-delay systems," Sys. Ctrl. Lett., vol. 55, pp. 1006-1014, 2006.

[22] N. Bekiaris-Liberis and M. Krstic, "Stabilization of strict-feedback linear systems with delayed integrators," Automatica, vol. 46, no. 11, 1902-1910, 2010

[23] N. Bekiaris-Liberis and M. Krstic, "Delay-adaptive feedback for linear feedforward systems," Sys. Ctrl. Lett., vol. 59, pp. 277-283, 2010.

[24] N. Bekiaris-Liberis and M. Krstic, "Compensation of time-varying input and state delays for nonlinear systems," ASME J. Dyn. Sys. Meas. Ctrl., vol. 134 , no. 1,2011

[25] N. Bekiaris-Liberis and M. Krstic, "Compensation of state-dependen input delay for nonlinear systems," IEEE Trans. Autom. Ctrl., vol. 58, no. 2, pp. 275-289, 2013.

[26] M. Krstic, "Input delay compensation for forward complete and feedforward nonlinear systems," IEEE Trans. Autom. Ctrl., vol. 55, no. 2, 287-303, 2010

[27] R. Kamalapurkar, N. Fischer, S. Obuz, and W. E. Dixon, "Time-varying input and state delay compensation for uncertain nonlinear systems," arXiv preprint, arXiv:1501.03810, 2015.

[28] H. T. Dinh, N. Fischer, R. Kamalapurkar, and W. E. Dixon, "Output feedback control for uncertain nonlinear systems with slowly varying input delay," In Proc. Amer. Ctrl. Conf., pp. 1745-1750, 2013.

[29] N. Bekiaris-Liberis and M. Krstic, "Robustness of nonlinear predictor feedback laws to time- and state-dependent delay perturbations," Automatica, vol. 49, no. 6, pp. 1576-1590, 2013.

[30] M. Krstic, Delay Compensation for Nonlinear, Adaptive, and PDE Systems. Boston, MA: Birkhauser, 2009.

[31] G. Goodwin, P. Ramadge, P. Caines, "Discrete-time multivariable adaptive control," IEEE Trans. Autom. Ctrl., vol. 25, no. 3, pp. 449-456, 1980

[32] K. P. V. Kokotovic, Foundations of Adaptive Control, Springer-Verlag, New York, 1991

[33] S. Akhtar and D. S. Bernstein, "Lyapunov-stable discrete-time model reference adaptive control," Int. J. Adaptive Ctrl. Signal Proc., vol. 19, no. 10, pp. 745-767, 2005

[34] S. Akhtar and D. S. Bernstein, "Logarithmic Lyapunov functions for direct adaptive stabilization with normalized adaptive laws," Int. J. Ctrl., vol. 77, no. 7, pp. 630-638, 2004

[35] D. Bresch-Pietri, M. Krstic, "Adaptive trajectory tracking despite unknown input delay and plant parameters," Automatica, vol. 45, no. 3, pp. 2074 2081, 2009.

[36] K. Abidi and Y. Yildiz, "Discrete-time adaptive posicast controller for uncertain time-delay systems," In Proc. AIAA Guid. Nav. Ctrl. Conf., AIAA Paper 2011-5788, Portland, ORpp. 1-11, 2011.

[37] K. Abidi and Y. Yildiz, "On the discrete adaptive posicast controller," Proc. $12^{\text {th }}$ IFAC Workshop Time-Delay Syst., Ann Arbor, MIpp. 422-426, 2015

[38] K. Abidi and J.-X. Xu, Advanced Discrete-Time Control: Designs and Applications, New York Springer, 2015.

[39] K. Abidi and X.-J. Xu, "On the discrete-time integral sliding-mode control," IEEE Trans. Autom. Ctrl., vol. 52, no. 4, pp. 709-715, 2007.

[40] T. Nguyen, W.-C. Su, and Z. Gajic, "Output feedback sliding mode control for sampled-data systems," IEEE Trans. Autom. Ctrl., vol. 55, no. 7, pp. 1684-1689, 2010.

[41] K. Abidi, "A robust discrete-time adaptive control approach for systems with almost periodic time-varying parameters," Int. J. Rob. Nonlin. Ctrl., vol. 24 , no. 1, pp. 166-178, 2014.

[42] J. H. Blakelock, Automatic Control of Aircraft and Missiles, Wiley, New York2nd edition, 1991 